Vol. 30, julho 2014, http://dx.doi.org/10.5380/dma.v30i0.34073

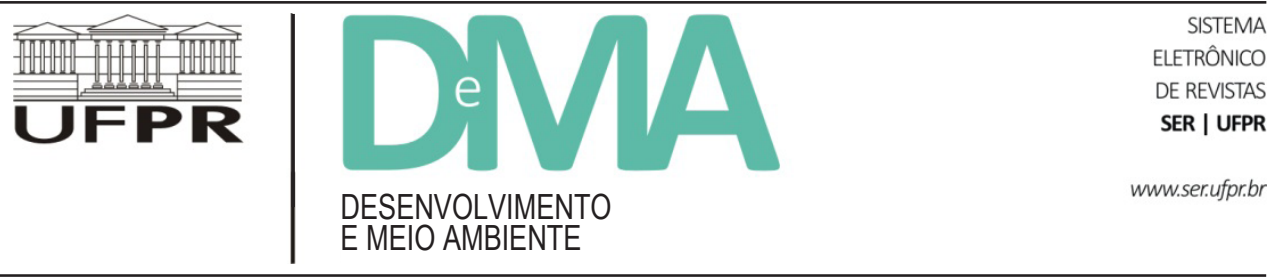

\title{
Análisis del Uso del Bioetanol como Medida de Mitigación y su Impacto en el Sector Hídrico: Estudio en una Región Cañera en México
}

\section{Análise do uso do bioetanol como medida de mitigação e seu impacto no setor hídrico: estudo em uma região canavieira no México}

\section{Analysis of the Use of Bioethanol as a Mitigation Measure and its impact on the Water Sector: a Study in a Sugarcane Region in Mexico}

\author{
Inés Josefina Gonzalez NAVARRO ${ }^{1}$, Blanca Elena JIMÉNEZ ${ }^{2}$, María Eugenia HARO ${ }^{3}$, Nidya APONTE \\ ${ }^{1}$ M. en C. en Sistemas Ambientales (ITESM, México). Enlace Institucional Instituto de Ingeniería, Universidad Nacional Autónoma de México \\ (UNAM). E-mail: INavarroG@iingen.unam.mx \\ ${ }^{2}$ Dra. en Tratamiento de Agua y Agua Residual (Institut National des Sciences Appliquées, Francia). Enlace Institucional Instituto de Ingeniería, \\ Universidad Nacional Autónoma de México (UNAM). E-mail: BJimenezC@iingen.unam.mx \\ ${ }^{3}$ M. en I. en Ingeniería Ambiental (UNAM, México). Enlace Institucional Instituto de Ingeniería, Universidad Nacional Autónoma de México \\ (UNAM).E-mail: mar577@yahoo.com.mx
}

${ }^{4}$ M. en I. en Ingeniería Ambiental (UNAM, México). Enlace Institucional Instituto de Ingeniería, Universidad Nacional Autónoma de México (UNAM). E-mail: naponteh@gmail.com

Artículo: Recibido el 25 de octubre 2013; versión final aceptada el 18 de abril 2014.

RESUMEN El uso de biocombustibles en el transporte es una medida de mitigación para reducir emisiones de gases de efecto invernadero; sin embargo pueden tener efectos ambientales negativos. Esta investigación analiza el impacto actual y futuro de la producción de etanol a partir de caña de azúcar en los recursos hídricos de México. Se estimó la huella hídrica, la disponibilidad y demanda de agua en una región cañera del país, con base en un balance hídrico y las condiciones climáticas locales. Los resultados mostraron que la huella hídrica histórica (1980-2007) de la caña de azúcar fue de $104.9 \mathrm{~m}^{3} /$ ton considerando el empleo de riego rodado. Sin embargo, en la región estudiada se utilizan tres sistemas de riego (rodado, aspersión y goteo), por lo que la huella hídrica varía entre 58.7 y $73.4 \mathrm{~m}^{3} /$ ton, que indican el ahorro del $20 \%\left(33 \mathrm{Mm}^{3}\right)$ de agua. La disponibilidad de agua en la región se estimó en $255 \mathrm{Mm}^{3} /$ año con un índice de estrés de $60 \%$, que indica un grado de presión alto sobre el recurso hídrico. Para los escenarios futuros de cambio climático se estimó un incremento del 3\% en la huella hídrica; sin embargo, el balance hídrico mostró que este incremento relativamente bajo tendrá un impacto significativo en la demanda de agua. México cuenta con una disponibilidad 18 veces menos que Brasil 
y casi 7 veces menos que Estados Unidos, de manera que la alternativa de producir etanol requiere del estudio de cada una de las regiones cañeras para evaluar sus ventajas y desventajas.

Palabras clave: biocombustibles; estrés hídrico; mitigación.

RESUMO O uso de biocombustíveis nos transportes é uma medida de mitigação para reduzir as emissões de gases do efeito estufa; entretanto, pode ter efeitos ambientais negativos. Esta pesquisa analisa os impactos atual e futuro da produção de etanol a partir da cana-de-açúcar nos recursos hídricos do México. Estimou-se a pegada hídrica, a disponibilidade e a demanda de água em uma região canavieira do país com base no balanço hídrico e nas condições climáticas locais. Os resultados mostraram que a pegada de água histórica (1980-2007) da cana-de-açúcar foi de 104,9 m³/t, considerando o uso de irrigação por sulco. No entanto, na região estudada são utilizados três sistemas de irrigação (por sulco, aspersão e gotejamento), razão pela qual a pegada de água varia entre $58,7 \mathrm{e} 73,4 \mathrm{~m}^{3} / \mathrm{t}$, o que indica uma economia de $20 \%\left(33 \mathrm{~mm}^{3}\right)$ de água. A disponibilidade de água na região foi estimada em $255 \mathrm{Mm}^{3} /$ ano, com um índice de estresse de $60 \%$, indicando um elevado grau de pressão sobre os recursos hídricos. Para cenários futuros de mudança climática, estimou-se um aumento de $3 \%$ na pegada de água; no entanto, o balanço hídrico mostrou que este aumento relativamente pequeno terá um impacto significativo na demanda de água. O México tem uma disponibilidade 18 vezes menor do que a do Brasil e quase sete vezes menor do que a dos EUA, de modo que a alternativa de produzir etanol requer o estudo de cada uma das regiões canavieiras para avaliar suas vantagens e desvantagens.

Palavras-chave: biocombustíveis; estresse hídrico; mitigação.

ABSTRACT The use of biofuel in transport is a mitigation measure to reduce emissions of greenhouse gases, but may have negative environmental effects. This research analyzes the current and future impact of the production of ethanol from sugar cane in Mexico's water resources. The water footprint, availability, and demand of water in a sugarcane region of the country were estimated, based on a water balance and local climatic conditions. The results showed that the historical water footprint (1980-2007) of sugarcane was $104.9 \mathrm{~m}^{3} / \mathrm{ton}$, considering the use of furrow irrigation. However, in the studied region three irrigation systems (furrow, spraying and drip) are used, so the water footprint varies between 58.7 and $73.4 \mathrm{~m}^{3} /$ ton, indicating savings of $20 \%\left(33 \mathrm{Mm}^{3}\right)$ of water. The availability of water in the region was estimated at $255 \mathrm{Mm}^{3} /$ year with a stress index of $60 \%$, indicating a high degree of pressure on the water resource. For future scenarios of climate change it was estimated that the water footprint increased 3\%; however, the water balance showed that this relatively small increase will have a significant impact on the water demand. Mexico has water availability 18 times smaller than Brazil and almost 7 times smaller than the U.S., so the alternative of producing ethanol requires the study of each of the sugarcane regions to assess their advantages and disadvantages.

Keywords: biofuel; water stress; mitigation.

\section{Introducción}

Los tipos de biocombustibles básicos, conocidos como de primera generación, son el bioetanol y biodiesel producidos a partir de cultivos comerciales. El etanol se produce a partir de azúcares y almidones, los cuales se extraen de biomasa de cultivos como el maíz, la caña de azúcar, yuca, sorgo y remolacha azucarera. El biodiesel se elabora a partir de aceites vegetales que se extraen de plantas oleaginosas como la palma, así como de grasas animales, aceites y grasas recicladas.

Las ventajas del uso de los biocombustibles en el transporte para la reducción de la emisión de gases de efecto invernadero (principalmente $\mathrm{CO}_{2}$ ) y para reducir el alto consumo de combustibles fósiles, como parte de una estrategia de seguridad energética, han sido ampliamente documentadas (SENER, 2006; Coviello et al., 2008). Los estudios disponibles hasta ahora se han enfocado en su mayoría al balance neto de energía o en el impacto en 
el efecto invernadero neto (Hofstrand, 2009; Shapouri et al., 2010). Sin embargo, desde el punto de vista de una estrategia de seguridad hídrica, los estudios aún muestran limitaciones en el conocimiento y discusión sobre el impacto de la producción de biocombustibles y la crisis del agua en muchas naciones. En este sentido, los resultados de la investigación que se reportan en este artículo buscan contribuir al conocimiento y análisis de los posibles efectos en el recurso agua, en el contexto de la producción de bioetanol a partir de caña de azúcar, en un país como México con baja disponibilidad de agua.

\section{La producción de bioetanol y el consumo de agua}

La producción de etanol combustible a partir de diversos cultivos ha crecido significativamente en los últimos años. Para 2007 se reportó una producción mundial total de 39 mil millones de litros (REN21, 2008), con una producción de los principales productores de etanol combustible en el mundo de 18.3 mil millones de litros en EE.UU. y en Brasil de 17.5 mil millones de litros. Para el año 2011, la producción total se incrementó a 84.360 mil millones de litros (Maluenda, 2011), aproximadamente, con 21.637 mil millones en Brasil y 54.765 mil millones de litros en EE.UU. (GRFA, 2011); la Unión Europea contribuyó con 4.429 mil millones de litros. En Europa Occidental, el etanol se produce en menor escala a partir de remolacha y trigo con un costo mayor, dos a cuatro veces más, que el costo del etanol brasileño (SENER, 2010).

Las diferencias entre los dos principales productores de bioetanol para el transporte, EE.UU. y Brasil, además de producirlo a partir de maíz y caña de azúcar, respectivamente, Brasil tiene más plantas en operación (335 vs 97), rendimientos más altos (66.2 ton/ha vs 8.4 ton/ha) y mayor productividad $(6,880 \mathrm{~L} /$ ha vs $3,000 \mathrm{~L} /$ ha). En 2006, Brasil destinó solo 1\% de su área cultivable para producir etanol, mientras que Estados Unidos destinó un 3.7\% del total de las tierras cultivables; además, el mayor porcentaje de la producción brasileña se destina para el transporte (40\% vs $3.8 \%$ ) y con menores costos de producción (0.22 dlls/L vs $0.40 \mathrm{dlls} / \mathrm{L})$ (Jank et al., 2007; Goettemoeller \& Goettemoeller, 2007).
Esos datos ejemplifican que la estrategia actual de desarrollo de los biocombustibles se enfoca a una intensa explotación de monocultivos de granos comerciales como la caña de azúcar y el maíz. De hecho, uno de los argumentos que han apoyado el rápido crecimiento del sector de biocombustibles es que son una alternativa de energía renovable a la dependencia de los combustibles fósiles. Sin embargo, los biocombustibles no son estrictamente renovables como lo son las fuentes de energía solar o eólica, ya que dependen para su producción de recursos finitos, en cantidad y calidad apta de tierra y agua. Además, la práctica agrícola del monocultivo lleva asociada el uso intensivo de pesticidas, fertilizantes y el uso de agua. De manera que el acelerado crecimiento de la producción de biocombustibles no sólo sugiere la posibilidad de futuros conflictos en la competencia por el uso de la tierra y el agua, sino que hay disponibles algunos estudios que analizan estos posibles eventos.

En este sentido, el estudio de Fresco (2007) discute principalmente el dilema entre la producción de biomasa para obtener alimentos o biocombustibles, sin embargo aborda brevemente las implicaciones en el recurso agua. No obstante, en otros estudios se reporta que actualmente la producción agrícola de biomasa para la alimentación requiere de cerca del $86 \%$ del agua dulce empleada a nivel mundial (Hoekstra, 2007). En muchas partes del mundo, este uso compite ya fuertemente con otros usos, como el abastecimiento urbano o el industrial. Un aumento en la demanda de agua para generar alimentos en combinación con un cambio de la energía fósil a energía de biomasa ejercerá una presión adicional sobre el recurso agua. Basado en esto, las Naciones Unidas señalan que para el año 2050 habrá motivos de preocupación por la falta de agua en varios países y regiones con recursos hídricos limitados (Gerbens-Leenes et al., 2009), y que esta situación se puede agravar a causa del uso de biocombustibles.

De acuerdo con un estudio reciente de la CEPAL (Saulino, 2011) el aumento en la producción de biocombustibles requerirá incrementar considerablemente la cantidad de biomasa producida a través de la intensificación del uso de la tierra y la expansión de las áreas cultivadas, y advierte que estos cambios en el uso de la tierra pueden tener un impacto significativo en los recursos hídricos. En ese estudio se señala que la pro- 
ducción de biocombustibles a base de cultivos bajo riego requiere mayores volúmenes de agua en comparación con la agricultura de temporal, cuya influencia en la disponibilidad de recursos hídricos a nivel local y regional es normalmente mucho menor. Por ello recomienda que debiera evaluarse a nivel local, regional y de cuencas las diferencias en la demanda y productividad del agua entre las distintas combinaciones de cultivo, región geográfica y la tecnología utilizada en la producción de biocombustibles.

Sin embargo, en pocos estudios (Varghese, 2007; Hughes et al., 2007) se documentan los impactos por la producción de los biocombustibles en la disponibilidad de agua a nivel local o regional. Un ejemplo es el estudio realizado por la FAO para evaluar la viabilidad de la producción de biocombustibles en una región andina del Perú (Ramos, 2010); estudios similares se han realizado en Tailandia y Tanzania (Felix \& Rosell, 2010).

En EE.UU. se han realizado estudios para estimar las necesidades de agua para la producción primaria de energía, incluyendo estimaciones para carbón, petróleo, gas y biomasa (Elcock, 2008) e identifican a los biocombustibles como los mayores consumidores de agua dentro del sector energético. Efectivamente, varios estudios se han realizado sobre el impacto de la producción de etanol a partir del cultivo de maíz en EE.UU. donde analizan los posibles efectos por la expansión de la producción de biocombustibles y la reducción en el agua disponible (Roberts et al., 2007; NRC, 2008). Un caso se refiere a la sobreexplotación del acuífero de Ogallala, identificado como uno de los más grandes del mundo; este acuífero es la fuente de suministro de la agricultura de riego en las grandes planicies del sur de EE.UU. En las zonas donde se ha observado la mayor reducción en el nivel freático operan 5 plantas con una producción anual de 71.5 millones de galones de etanol y están en construcción 9 más con una capacidad anual de 639 millones de galones. Se estima que las nuevas plantas demandarán anualmente un volumen de agua equivalente a 2.6 billones de galones y si se incrementa la extensión de riego para la producción de maíz, se podrá incrementar la demanda entre 59 y 210 billones de galones por año, agravando aún más el actual nivel de estrés hídrico en la región (Roberts et al., 2007).
Otro estudio realizado en EE.UU. reporta que la mayoría de la producción de maíz para la producción de bioetanol se realiza bajo riego y el agua subterránea es la principal fuente de abastecimiento (Chiu et al., 2009). Los resultados reportados del consumo de agua presentan una gran variabilidad ya que hay sitios donde equivale a $5 \mathrm{~L} / \mathrm{L}$ etanol con un consumo máximo de $2,138 \mathrm{~L} / \mathrm{L}$ etanol. El estudio muestra que la mayor demanda de agua en 2007 corresponde al agua para riego; por ejemplo, en el estado de Iowa, se consume en promedio 17,288 millones de litros de agua para riego y 24,745 millones de litros en el proceso para obtener etanol; en el estado de Minnesota la demanda para riego es de 34,589 millones de litros anuales, mientras que en el proceso se emplean 8,286 millones de litros; en California la demanda para riego se incrementa hasta 549,240 millones de litros de agua, mientras que en el proceso se emplean solo 929 millones de litros. Estos datos ejemplifican la variabilidad regional que no se consideró en otros estudios previos que reportaban un uso entre 263 y 784 litros de agua por cada litro de etanol (Pimentel, 2003; de Fraiture et al., 2008; NRC, 2008). Por ello es importante señalar que los requerimientos de agua dependen de la región, las condiciones climáticas, la variedad de maíz y la eficiencia en las destilerías.

En el caso de Brasil, segundo productor de bioetanol a partir de caña de azúcar, la producción ha sido tradicionalmente de temporal para la cual se estima que el consumo de agua de lluvia varía entre 1,500 - 2,500 mm/año. Sin embargo, Moreira (2007) reporta que está presente la tendencia a abrir campos de cultivo en zonas de riego. En general se reconoce que hay un uso eficiente de agua, tanto en la producción de caña como en las destilerías o ingenios donde se procesa la melaza para producir etanol y tiene un consumo promedio de agua de $21 \mathrm{~m}^{3}$ por tonelada de caña.

Las evidencias hasta aquí presentadas conducen a explicar cómo se usa el agua y principalmente a cómo estimar el volumen empleado en toda la cadena de producción de los biocombustibles. La producción de biocombustibles requiere del uso de agua en dos etapas primordiales: en el crecimiento de las materias primas en los campos agrícolas y en el proceso de producción industrial de los biocombustibles. Si se considera sola- 
mente el uso del agua en los ingenios o destilerías, donde se procesa la materia prima para obtener los biocombustibles, la producción de biocombustibles da la impresión de tener un impacto mínimo sobre el consumo de agua, especialmente cuando se les compara con las plantas convencionales de producción de derivados del petróleo (Gleick, 2000). Sin embargo, la situación cambia cuando se estima el total de agua usada por galón de etanol producido ya que el mayor consumo está asociado a la demanda para la producción de la materia prima.

El consumo de agua en la producción agrícola de la materia prima varía de acuerdo a si la selección de las zonas de cultivo corresponde a: a) conversión de la vegetación natural local a zona agrícola, b) aumento de las prácticas actuales de producción del cultivo seleccionado, c) introducción de cultivos diferentes en suelo agrícola existente, o d) en el uso de la vegetación natural existente como materia prima. A esta variable que corresponde al tipo de uso del suelo previo, se suman otros factores importantes que influyen en la variación del volumen de agua necesario para la producción de la materia prima, tales como: el tipo y método de cultivo, las características agroclimáticas específicas, la evapotranspiración en los diferentes estadios del crecimiento del cultivo y la demanda de agua para irrigación. Este último puede ser un factor relevante en aquellas zonas donde se cuente con poca agua disponible y en donde los sistemas de riesgo operen con bajas eficiencias.

Con respecto a la etapa de la producción de bioetanol a partir de la caña de azúcar en los ingenios, esta consiste de tres fases: extracción de la melaza, fermentación y destilación. De la caña de azúcar se extrae la melaza, que contiene entre $35 \%$ y $40 \%$ de azúcares, para luego ser diluida y combinada con levaduras. Con la fermentación de dicha solución se genera dióxido de carbono y licor fermentado (técnicamente denominado mieles); este último, que sólo contiene 10\% de alcohol, se somete a una serie de destilaciones sucesivas con el fin de obtener un alcohol de mayor pureza. En estos procesos, se presenta un elevado consumo de agua principalmente en el lavado de la caña tan pronto llega de los campos de cultivo, así como en la generación de vapor para las diferentes etapas del proceso.

\section{Huella hídrica de los biocombustibles}

El concepto para evaluar los requerimientos de agua para la producción de un producto se conoce como la huella hídrica (Hoekstra \& Hung, 2002; Hoekstra \& Chapagain, 2007); ésta se define como el volumen total de agua usado para la producción de bienes y servicios. A partir del año 2002 el profesor holandés Arjen Y. Hoekstra (Hoekstra \& Hung, 2002) desarrolla el concepto de huella hídrica. La huella hídrica puede estimarse en términos de la fuente de agua y se identifican como la huella hídrica azul, verde o gris.

La huella de agua azul es el volumen de agua dulce consumida de los recursos hídricos disponibles del planeta (agua superficial y/o subterránea) la cual corresponde a estimar la empleada a través de algún sistema de irrigación. La huella del agua verde es el volumen de lluvia almacenada en el suelo como humedad que corresponde al aprovechamiento de agua en las plantaciones de temporal. La huella de agua gris se refiere al volumen de agua que se requiere para asimilar la carga de contaminantes que rebasan las concentraciones naturales del lugar y las normas de calidad del agua establecidas (Hoekstra et al., 2009).

Actualmente es posible encontrar entre la bibliografía internacional relacionada al tema estudios que aplican el concepto de la huella hídrica. Por ejemplo, Van Meekeren (2008) analizó la huella hídrica de los biocombustibles con el objeto de cuantificar la relación entre el agua dulce y los biocombustibles. Hoekstra \& Chapagain (2008) identifican la estrecha relación que la globalización impone entre el manejo de agua y el comercio internacional a partir del análisis del uso del recurso hídrico a nivel de países. Mekonnen \& Hoekstra (2010) estimaron la huella hídrica de varios cultivos (trigo, maíz, arroz, manzana, soya, caña de azúcar, café y algodón) con base en datos de producción del periodo 1996-2005; encontraron que el cultivo de trigo, arroz y maíz requiere de un volumen mayor de agua para su crecimiento que el cultivo de la caña de azúcar (Fig. 1).

Además Mekonnen \& Hoekstra (2010) estimaron la huella hídrica global bajo condiciones de riego y de temporal; la Tabla 1 muestra sus resultados para los principales cultivos que se producen bajo riego (huella 


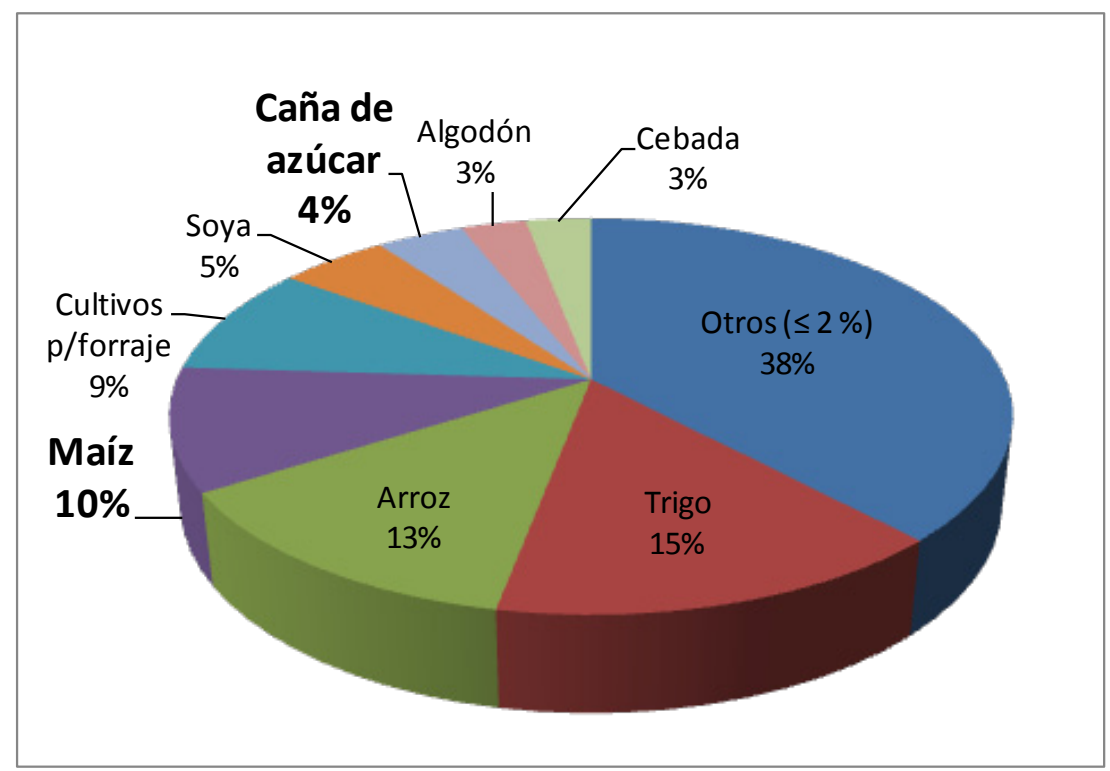

FIGURA 1 - Contribución de varios cultivos a la huella hídrica.

(Elaborado con datos de Mekonnen \& Hoekstra, 2010)

hídrica azul) y se observa que la demanda de riego para la caña de azúcar es menor al 50\% de la requerida para el cultivo de maíz.

TABLA 1 - Huella hídrica de diferentes cultivos bajo riego

\begin{tabular}{lc}
\hline \multicolumn{1}{c}{ Cultivo } & $\begin{array}{c}\text { Huella hídrica azul } \\
{\left[\mathbf{m}^{3} / \text { ton }\right]}\end{array}$ \\
\hline Caña de azúcar & 104 \\
Maíz & 294 \\
Arroz & 464 \\
Trigo & 926 \\
Algodón & 2227 \\
\hline
\end{tabular}

FUENTE: Mekonnen \& Hoekstra, 2010.

En el estudio realizado por Gerbens-Leenes et al. (2009) se analizó la huella hídrica de 13 cultivos: cebada, yuca, maíz, papa, colza, arroz, centeno, sorgo, soya, remolacha azucarera, caña de azúcar, trigo y jatropha. Éstos en conjunto representan el $80 \%$ de la producción a nivel mundial, siendo la más importante la de caña de azúcar con 1,258 millones de ton/año, seguida por el maíz, trigo y arroz con cerca de 600 millones de ton/año cada uno. El estudio incluye estimaciones del consumo total de agua en la producción de biocombustibles y reportan que la huella hídrica del bioetanol es menor que la del biodiesel (29.7 kJoule/g y 37.7 kJoule/g, respectivamente). Asimismo, identifican que la huella hídrica de los biocombustibles muestra una gran variación que depende de tres factores: el cultivo utilizado, el clima del lugar de producción y la práctica agrícola. Además concluyen que la huella hídrica de los biocombustibles es muy grande en comparación con otras formas de generar energía, lo cual confirma lo reportado en otros estudios.

En otro estudio, Gerbens-Leenes et al. (2009a) también estiman la huella hídrica total de los principales países productores de caña de azúcar en el mundo (Fig. 2), independientemente de si parte de esa producción se emplea para producir biocombustibles. En la figura se reporta la estimación de la huella hídrica como un litro de agua por litro de etanol con un promedio ponderado global de $2,855 \mathrm{~L}_{\text {agua }} / \mathrm{L}_{\text {etanol }}$; se observa que países con menores requerimientos, como Perú y Egipto, dependen del agua de riego (huella azul), mientras que Indonesia y Filipinas dependen del agua de lluvia (huella verde). 


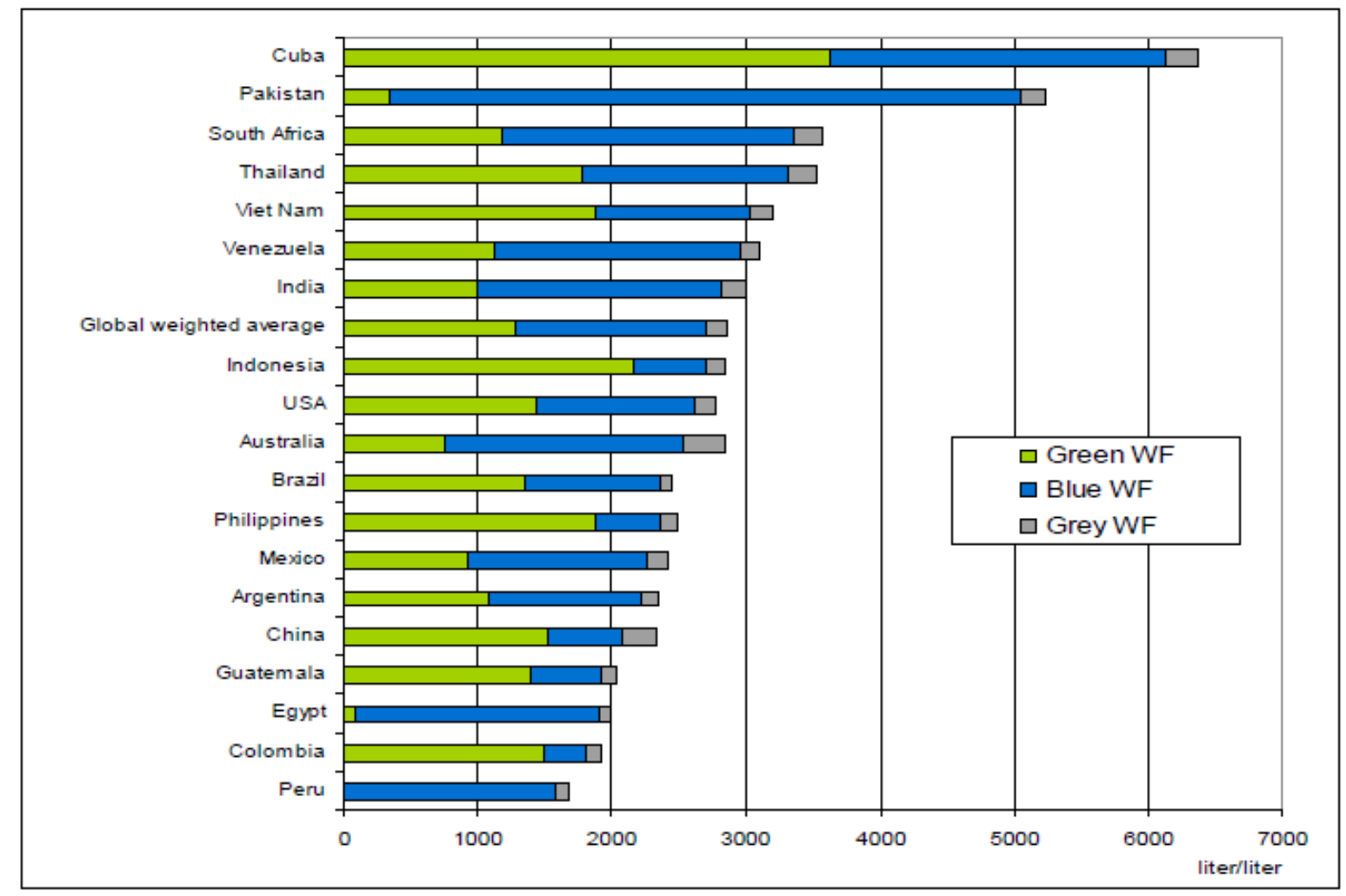

FIGURA 2 - Huella hídrica del etanol de caña de azúcar en los principales países productores.

FUENTE: Gerbens-Leenes et al., 2009a.

\subsection{Estimación de la huella hídrica}

La metodología propuesta por Hoekstra et al. (2009) y formalmente compilada en un manual, comprende la estimación de la huella hídrica del proceso completo de crecimiento de un cultivo ( $\mathrm{HH}$ en $\mathrm{m}^{3} /$ ton). Este procedimiento, para el cálculo de la huella hídrica, se basa en estimar la cantidad de agua que se requiere para el crecimiento de un cultivo (CWU en $\mathrm{m}^{3}$ ) ha) dividido entre el rendimiento del cultivo ( $\mathrm{Y}$ en ton/ ha). El volumen de agua que requiere el cultivo para su crecimiento (CWU) se calcula a partir de la estimación de la evapotranspiración (ET en $\mathrm{mm}$ ) desde el primer día de la siembra hasta el día de la cosecha, considerando las condiciones agroclimáticas del sitio en estudio (Ecuación 1). Esta evapotranspiración diaria (ET) se calcula a partir de la estimación de la evapotranspiración de referencia del cultivo ( $\mathrm{ET}_{0}$ en $\mathrm{mm}$ ), multiplicada por el factor del cultivo $\left(\mathrm{K}_{\mathrm{c}}\right.$ ) para las diferentes etapas de su ciclo de crecimiento (Brouwer \& Heibloem, 1986); el parámetro $\mathrm{K}_{\mathrm{c}}$ representa características tales como la altura del cultivo, la cobertura del suelo y el albedo, aspectos que distinguen a un cultivo de la superficie agrícola del sitio en estudio (ALLEN et al., 1998).

$$
\mathrm{CWU}=10 \sum_{\mathrm{d}=1}^{1 \mathrm{gp}} \mathrm{ET}
$$

(Ecuación 1)

La componente más importante en la estimación de la huella hídrica de cualquier cultivo es la evapotranspiración de referencia diaria, tanto en términos del peso que tiene en el volumen de agua estimado, como en la información de datos agroclimáticos específicos al sitio 
en estudio que se requieren para su cuantificación. Esto se debe a que la evapotranspiración es la consideración conjunta de dos procesos diferentes: la evaporación y la transpiración. La evaporación es el proceso por el cual el agua es transferida desde la superficie terrestre hacia la atmósfera. Incluye tanto la evaporación de agua directamente desde el suelo o desde las superficies vegetales vivas o muertas (rocío, escarcha, lluvia interceptada por la vegetación), como las pérdidas de agua a través de las superficies vegetales, particularmente las hojas. La transpiración es el agua absorbida por medio de las raíces, que se transfiere a la atmósfera fundamentalmente a través de las estomas en las hojas. Por tanto, la evapotranspiración constituye la transferencia total de agua desde una superficie vegetal a la atmósfera (Domingo et al., 2002).

Cabe señalar, que la mayoría de los estudios que estiman la huella hídrica de los biocombustibles utilizan el método de Hoekstra et al. (2009), expuesto aquí brevemente. En algunos estudios se utiliza otro enfoque, como el que presentan Fingerman et al. (2010), quienes estiman el impacto en el recurso agua a partir del cálculo de la evapotranspiración de varios cultivos y la incorporan al análisis del ciclo de vida del consumo de agua del etanol. Además, en todos los estudios revisados para esta investigación se observó que al evaluar la huella hídrica para los biocombustibles, los autores calcularon la evapotranspiración de referencia de los cultivos $\left(\mathrm{ET}_{0}\right)$ con base en el método de Pennman-Monteith (Allen et al., 1998) recomendado por la FAO. La estimación de la evapotranspiración por este método permite caracterizar los efectos climáticos específicos del sitio de estudio ya que se calcula considerando parámetros como temperatura, radiación solar, velocidad del viento y humedad relativa.

Por ello, en el estudio de caso para México que se detalla en las siguientes secciones, se aplicó la metodología de Hoekstra et al. (2009) y el método de Pennman-Monteith (Allen et al., 1998) para las condiciones de producción que se presentaron durante la zafra 2010-2011; la huella hídrica estimada se compara posteriormente con la estimada para escenarios futuros con cambio climático, siguiendo los mismos procedimientos. Para poner en contexto el sitio de estudio seleccionado y los resultados obtenidos, se parte con una breve des- cripción de la producción cañera en el país y una rápida visión de la disponibilidad de agua.

\section{Regiones cañeras en México y la disponibilidad de agua}

En el país hay cinco regiones cañeras (Centro, Huastecas, Occidente, Sureste y Golfo) y 54 ingenios azucareros en operación. La actividad cañera se localiza en 15 estados (Veracruz, Jalisco, San Luis Potosí, Oaxaca, Chiapas, Tamaulipas, Nayarit, Puebla, Morelos, Quintana Roo, Tabasco, Sinaloa, Colima, Michoacán y en Campeche) donde se cultivaron 672,658 hectáreas para la zafra 2010-2011 (UNC, 2012), superficie que representa menos del $0.35 \%$ del territorio nacional.

De acuerdo a datos disponibles de la zafra 20052006 y 2006-2007, la producción de caña de azúcar en México depende del riego en aproximadamente $40 \%$ del área destinada a éste cultivo y el $60 \%$ es de temporal; con un rendimiento promedio de 89 ton/ha bajo riego y de 63 ton/ha para temporal (Sagarpa, 2009). En términos de la contribución a la producción de caña de azúcar que se cultiva bajo riego, destacan los estados de Veracruz, Jalisco, Tamaulipas y Sinaloa (Tabla 2).

TABLA 2 - Principales estados productores de caña de azúcar 19982008.

\begin{tabular}{lcccc}
\hline \multicolumn{1}{c}{ Estado } & $\begin{array}{c}\text { Riego } \\
\mathbf{( \% )}\end{array}$ & $\begin{array}{c}\text { Temporal } \\
\mathbf{( \% )}\end{array}$ & $\begin{array}{c}\text { Total } \\
\mathbf{( \% )}\end{array}$ & $\begin{array}{c}\text { RHA } \\
\text { dominante }\end{array}$ \\
\hline Veracruz & 24.6 & 50.1 & 38.4 & X \\
Jalisco & 21.9 & 2.6 & 12 & VIII \\
Tamaulipas & 9.8 & 2.1 & 5.8 & IX \\
Sinaloa & 9 & 0 & 4.3 & III \\
Morelos & 7.4 & 0 & 3.6 & IV \\
Chiapas & 6.5 & 2.9 & 4.7 & XI \\
San Luis Potosí & 4.3 & 9.2 & 6.9 & IX \\
Nayarit & 1.9 & 6.6 & 4.4 & VIII \\
Oaxaca & 0.5 & 12 & 6.6 & X \\
Tabasco & 0.1 & 6.5 & 3.5 & XI \\
\hline Total & 86 & 92 & 90.2 & \\
\hline Aportacion por & & & &
\end{tabular}

Aportación porcentual al total nacional FUENTE: Sagarpa, 2009. 


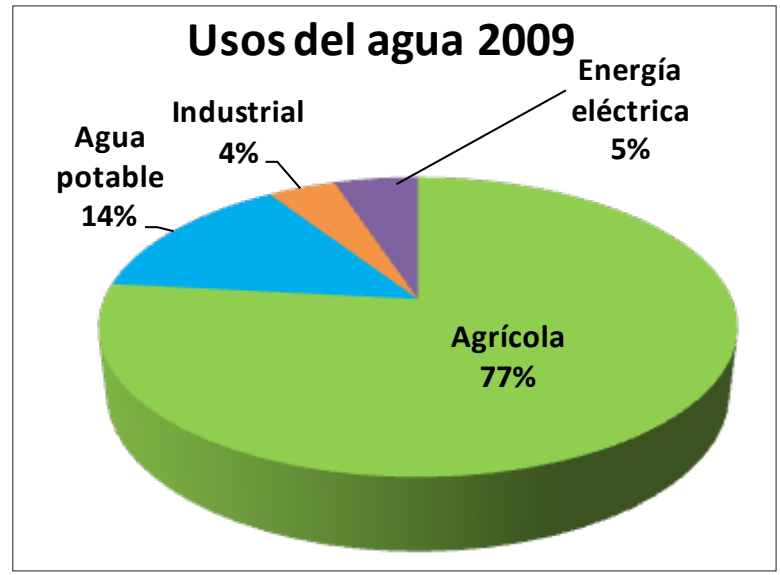

FIGURA 3 - Distribución de los volúmenes concesionados para los usos consuntivos del agua (CONAGUA, 2011).

En el contexto de esta investigación es importante recordar que el mayor volumen de agua disponible en el país se emplea en la actividad agrícola (76.7\%) (Fig. 3) (CONAGUA, 2011) a través de la irrigación de los terrenos en los distritos de riego y en las denominadas unidades de riego. Este porcentaje del volumen de agua empleado en la agricultura se asemeja al promedio global mundial que se reporta en 70\% (FAO, 2012a). Sin embargo, el volumen de agua que representa ese porcentaje depende de la disponibilidad de agua en cada país. Si comparamos los datos de disponibilidad promedio anual de los dos principales países productores de etanol en el mundo, con valores de 8,233,000 millones de $\mathrm{m}^{3} \mathrm{y}$ 3,069,000 millones de $\mathrm{m}^{3}$, para Brasil y EE.UU., respectivamente (FAO, 2012b), con el promedio nacional de 460,237 millones de $\mathrm{m}^{3}$ anuales (CONAGUA, 2011), se advierte que contamos en total con bastante menos agua y coloca al país en una situación de desventaja importante en el mercado de biocombustibles.

Además habrá que considerar que la disponibilidad natural media de agua per cápita en México ha ido disminuyendo con los años, pasando de $18,000 \mathrm{~m}^{3}$ por habitante al año en 1950 a menos de $4,000 \mathrm{~m}^{3}$ por habitante al año en el 2010 (CONAGUA, 2011). A causa del impacto del cambio climático, esta disponibilidad se verá afectada así como la demanda de la misma para los distintos usos consuntivos (Bates et al., 2008). CONAGUA estima que para el año 2030 el agua renovable per cápita alcanzará niveles cercanos o incluso inferiores a $\operatorname{los} 1,000 \mathrm{~m}^{3} /$ hab-año en algunas de las trece Regiones Hidrológicas Administrativas (RHA) en las que divide al país, lo que se califica como una condición de escasez grave (Tabla 3 ).

TABLA 3 - Disponibilidad natural media de agua en México.

\begin{tabular}{|c|c|c|c|}
\hline \multirow{2}{*}{$\begin{array}{c}\text { Región Hidrológica } \\
\text { Administrativa }\end{array}$} & \multirow{2}{*}{$\begin{array}{c}\text { Agua } \\
\text { renovable } \\
\text { promedio } \\
\text { (millones } \\
\mathbf{m}^{3} / \mathbf{a n ̃ o} \text { ) }\end{array}$} & \multicolumn{2}{|c|}{$\begin{array}{c}\text { Agua renovable per } \\
\text { cápita }\end{array}$} \\
\hline & & $\begin{array}{c}2010 \\
\left(m^{3} / \mathbf{h a b} /\right. \\
\text { año) }\end{array}$ & $\begin{array}{c}2030 \\
\left(\mathrm{~m}^{3} / \mathrm{hab} /\right. \\
\text { año) }\end{array}$ \\
\hline $\begin{array}{l}\text { I Península de Baja } \\
\text { California }\end{array}$ & 4,667 & 1,234 & 789 \\
\hline II Noroeste & 8,499 & 3,225 & 2,920 \\
\hline III Pacífico Norte & 25,630 & 6,475 & 6,754 \\
\hline IV Balsas & 21,680 & 2,033 & 1,948 \\
\hline V Pacífico Sur & 32,824 & 7,945 & 8,162 \\
\hline VI Río Bravo & 12,163 & 1,094 & 918 \\
\hline $\begin{array}{l}\text { VII Cuencas Centrales } \\
\text { del Norte }\end{array}$ & 7,898 & 1,873 & 1,729 \\
\hline $\begin{array}{l}\text { VIII Lerma-Santiago- } \\
\text { Pacífico }\end{array}$ & 34,533 & 1,633 & 1,469 \\
\hline IX Golfo Norte & 25,564 & 5,132 & 5,013 \\
\hline X Golfo Centro & 95,866 & 9,907 & 9,659 \\
\hline XI Frontera Sur & 157,754 & 23,637 & 21,041 \\
\hline $\begin{array}{l}\text { XII Península de } \\
\text { Yucatán }\end{array}$ & 29,645 & 7,151 & 5,105 \\
\hline $\begin{array}{l}\text { XIII Aguas del Valle } \\
\text { de México }\end{array}$ & 3,513 & 163 & 148 \\
\hline Total & 460,237 & 4,230 & 3,800 \\
\hline
\end{tabular}

FUENTE: CONAGUA (2011)

En la Figura 4 se puede observar la localización de las cuatro Regiones Hidrológicas Administrativas (RHA) con menor disponibilidad de agua per cápita en el 2010 (Tabla 3): la situación más grave se presenta en la Región XIII que comprende al DF y parte de los estados de Hidalgo, Tlaxcala y Estado de México; la Región VI que comprende a los estados de Chihuahua, Coahuila, Nuevo León y la parte norte de Tamaulipas; la Región I que abarca a toda la península de Baja California, y la Región VIII, conocida como Lerma-Santiago-Pacífico, con los estados de Jalisco, Colima, Aguascalientes, Guanajuato y parte de Nayarit y Michoacán. 


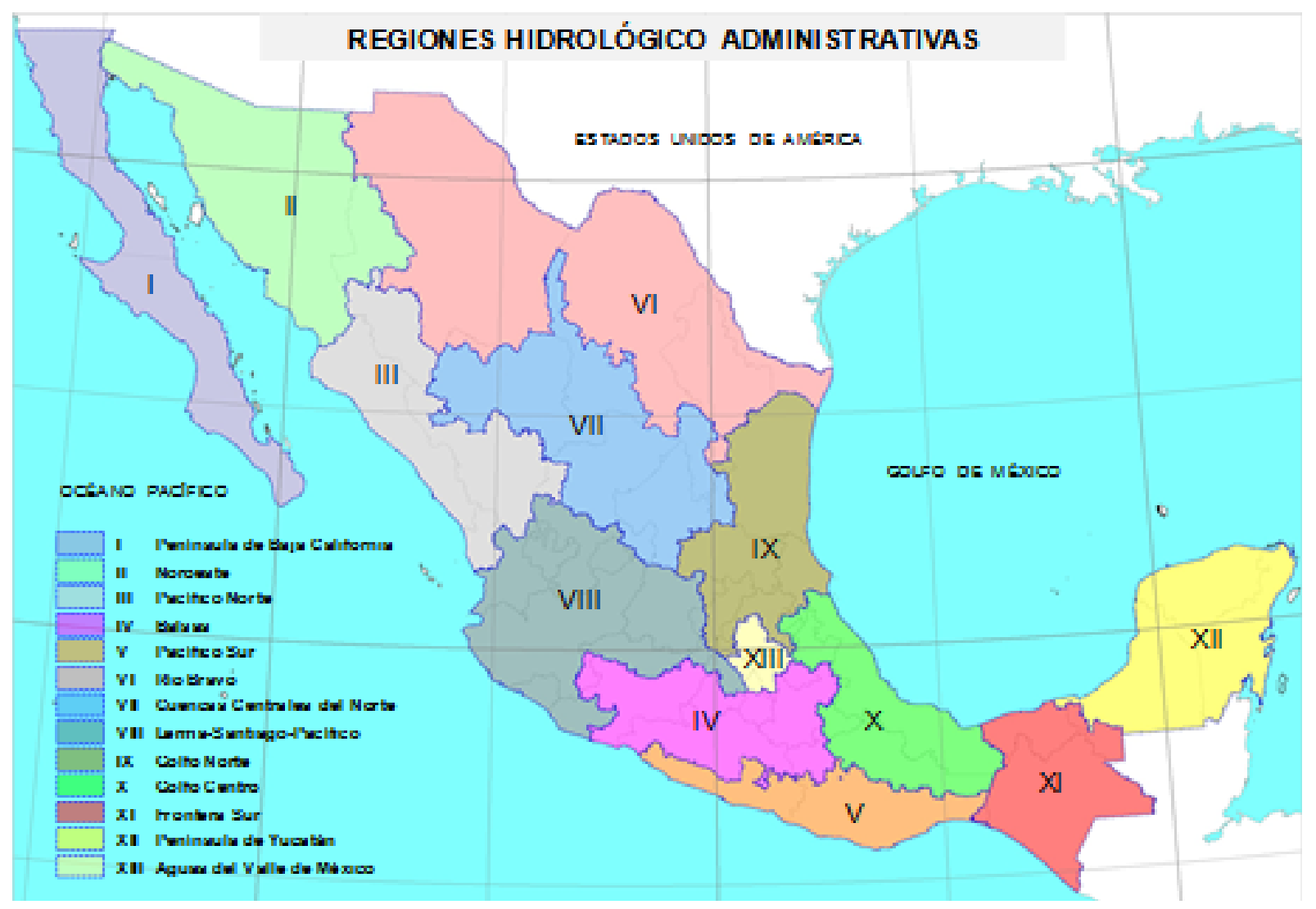

FIGURA 4 - Localización de la Regiones Hidrológicas Administrativas.

FUENTE: INEGI. Conjunto de Datos Geográficos de la Carta Hidrológica de Aguas Superficiales, 1:250,000.

En las Regiones Hidrológicas VIII y IV con baja disponibilidad de agua en el 2010 se localiza el $20 \%$ de la superficie cañera sembrada durante la zafra 2010 2011. En las regiones con mediana disponibilidad, que corresponde a la Regiones IX, XII y X, se encuentra el $70 \%$ de la superficie sembrada; y en la región con suficiente agua, la Región XI, sólo se cultivó el 10\% de la superficie total.

\section{Estudio de caso: Ingenio Tamazula}

Para la realización de esta investigación se logró tener contacto con la administración del Ingenio Tamazula A.C., perteneciente a la región cañera de Occidente.
Esto brindó la oportunidad de visitar sus instalaciones (Octubre 2011), recorrer la zona agrícola y obtener que proporcionaran la información disponible, solicitada para este estudio.

\subsection{Descripción del sitio de estudio}

La zona cañera asociada al ingenio azucarero Tamazula A.C. del Grupo Sáenz se localiza en el centro-oeste del país; en la región sur del Estado de Jalisco en el municipio Tamazula de Gordiano (Fig. 5) ubicado entre la latitud norte a 19 grados 41 minutos, longitud oeste a 103 grados y 15 minutos y a una altitud de 1,120 metros sobre el nivel del mar. Cuenta con una extensión 
territorial de 1,326.44 $\mathrm{km}^{2}$; colinda al norte con los municipios de Gómez Farías, Concepción de Buenos Aires, al noreste con Mazamitla, al este con Valle de Juárez, Manuel Diéguez, al oeste con Ciudad Guzmán, al sur oeste con Zapotiltic y al sur con Tecalitlan y Jilotlán de los Dolores.

El Municipio de Tamazula tiene una población de 109,166 habitantes; la región concentra el 1.62\% de la población total del Estado de Jalisco, correspondiéndole en razón de su superficie una densidad de 15.32 habitantes por $\mathrm{km}^{2}$ (CEA, 2011). El municipio presenta un clima templado cálido y semicálido, con temperatura media anual de $20.9^{\circ} \mathrm{C}$ y precipitación media anual de $897 \mathrm{~mm}$ (CEA, 2011). Sostiene principalmente comunidades vegetativas como selva mediana, pastizales, y bosques de pino y encino.

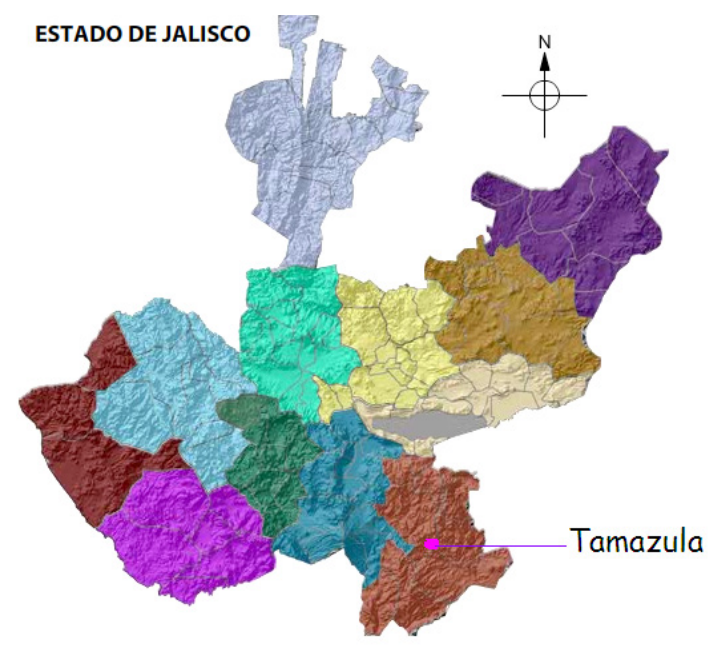

FIGURA 5 - Ubicación de Tamazula en el Estado de Jalisco (CEA, 2011).

FUENTE: INEGI. Conjunto de Datos Geográficos de la Carta Hidrológica de Aguas Superficiales. CEA Jalisco. Sistema Estatal de Información del Agua, 1:250,000.

La zona cañera abarca aproximadamente 15,500 hectáreas donde toda la producción se obtiene bajo el sistema de riego. Típicamente se aplica anualmente de febrero a septiembre, ya que el periodo de estiaje se presenta de octubre a junio y se observa una precipitación promedio histórica entre 0.2 y $32 \mathrm{~mm} / \mathrm{d}$. En los campos de cultivo hay infraestructura para aplicar el riego a través de tres sistemas: rodado (por inundación), por aspersión y por goteo (Fig. 6). La mayor parte de los terrenos agrícolas actualmente son regados por inundación (50\%); en los últimos años se ha incrementado el área regada por goteo de tal forma que cubre aproximadamente el $25 \%$ del área cultivada y un porcentaje igual por aspersión. Estos sistemas de riego, aspersión y por goteo, son más eficientes en el uso de agua porque permiten el ahorro entre 30 y $40 \%$ de agua, respectivamente (Narayanamoorthy, 2005).

El cambio tecnológico en los sistemas de irrigación también representa cambios en la fuente de abastecimiento de agua; ya que para los sistemas de riego por goteo y aspersión recurren a la extracción de agua subterránea, mientras que el riego rodado se realiza a través del bombeo de agua superficial procedente del Río Tamazula. La administración del ingenio Tamazula cuenta con infraestructura instalada en algunas secciones de ese río para represar el agua y bombear a los canales de riego, lo cual permite el manejo integral del agua superficial en la zona irrigada.

Además del nivel de tecnificación del sistema de riego en los campos de cultivo, la administración del ingenio tiene acceso a datos de temperatura y precipitación que obtienen de dos estaciones meteorológicas (Contla (14034) e Ingenio (14141)) localizadas dentro de las tierras cultivadas; así como con información a través de tres estaciones agro-climatológicas también ubicadas dentro de la zona de estudio (Tamazula, Tezontel y el Ovejo). Éstas cuentan con datos de precipitación, temperatura, velocidad del viento, radiación solar, humedad relativa, entre otros, y comenzaron a funcionar a partir de 2008.

Esta zona cañera destaca por lograr altos rendimientos agrícolas, del orden de 110 ton de caña por hectárea en promedio, comparados con el promedio nacional de 72 ton/ha (UNC, 2012). En términos de la capacidad industrial instalada, el ingenio cuenta con instalaciones para la producción de alcohol etílico, y produjo 1,944,155 L de alcohol, en el año 2010-2011, durante los 75 días que estuvo en operación. El volumen de producción de alcohol corresponde al uso del $30 \%$ promedio de las mieles producidas en el proceso de fabricación de azúcar. Es uno de los cinco ingenios del país que mantiene la producción de alcohol, ya que 


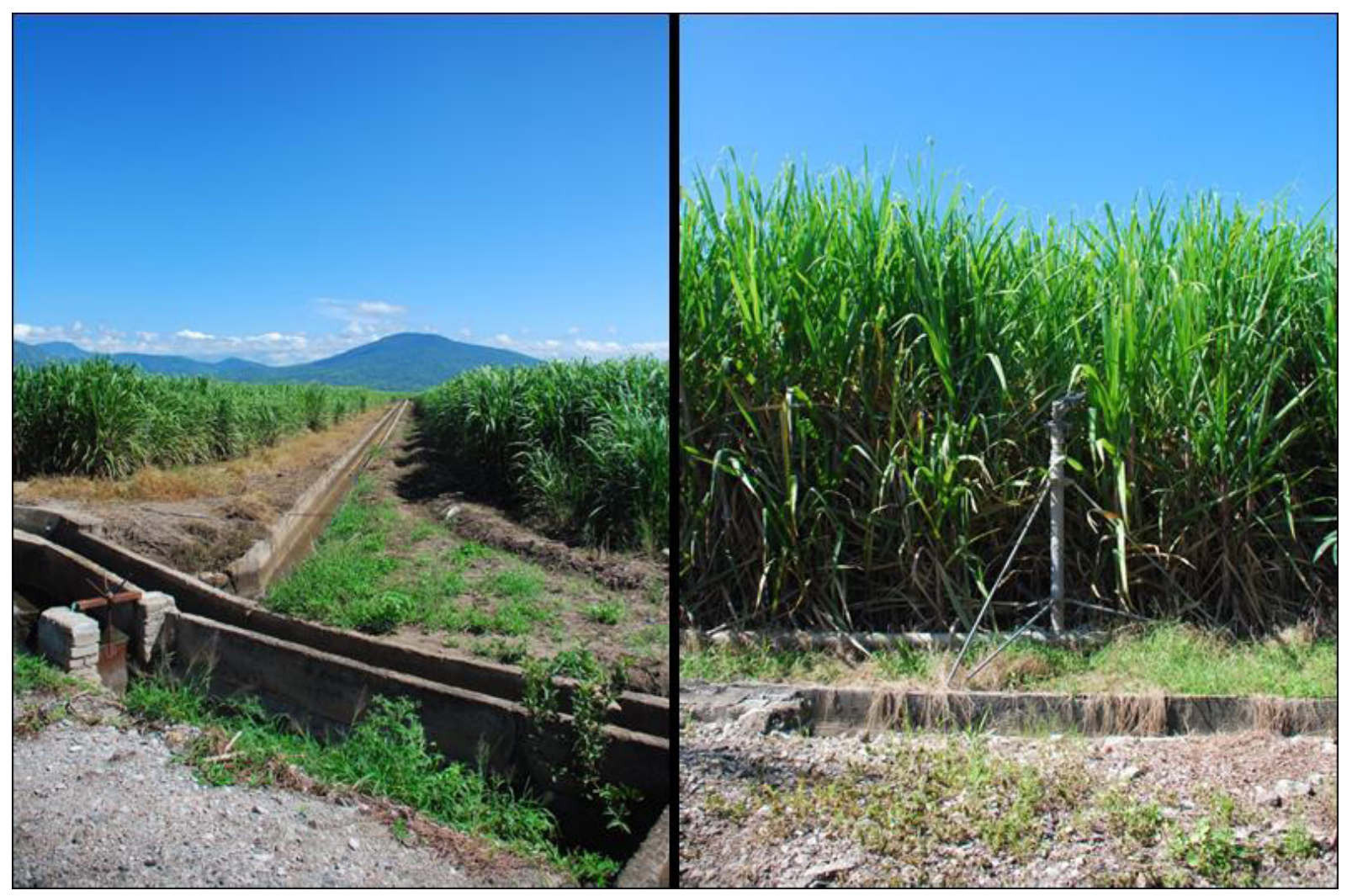

FIGURA 6 - Sistemas de riego rodado y por aspersión en la zona cañera de Tamazula.

otros cuentan con instalaciones pero no lo producen (UNC, 2012).

Otros aspectos a destacar del ingenio Tamazula son el área de investigación para el mejoramiento de semillas y el aprovechamiento de desechos. Cuenta con un laboratorio experimental donde desarrollan distintas variedades de caña y realizan pruebas para encontrar las más eficientes. El volumen de residuos y desechos que se producen en el ingenio son aprovechados casi en su totalidad y generan beneficios sociales, económicos y ambientales. Por ejemplo, el porcentaje de mieles que no se procesa tiene un importante mercado en la zona ya que se comercializa para la industria alimentaria; lo mismo ocurre con el volumen de melaza que se usa como alimento para el ganado. En términos de los beneficios ambientales, cabe señalar que las vinazas producidas en el ingenio son aprovechadas en la fertilización de los suelos agrícolas y cuentan además con instalaciones para la práctica de la lombricultura con el fin de utilizar el producto también como abono. Una reciente innovación en el ingenio fue la inauguración en 2011 de una planta de cogeneración de energía, que destaca al Grupo Sáenz como los primeros dentro del sector azucarero.

\subsection{Consumo de agua para la producción de bioetanol}

La estimación de la demanda de agua para la producción de bioetanol en el caso de estudio comprende el cálculo del agua necesaria para el cultivo de la caña de azúcar bajo las características específicas del sitio, así 
como el volumen de agua requerida en el ingenio para obtener el etanol a partir del procesamiento de las mieles. Estos cálculos de la huella hídrica se presentan para datos históricos del periodo de 1980 al 2007, y considerando proyecciones futuras de precipitación y temperatura bajo escenarios de cambio climático. Para las proyecciones futuras se estimó el valor que podría esperarse en los requerimientos de agua para el año 2020 y 2050.

A partir del análisis de los datos históricos, se estimaron las variables necesarias para el cálculo de la huella hídrica $(\mathrm{HH})$. La estimación de la evapotranspiración de referencia $\left(\mathrm{ET}_{0}\right)$ que es la variable más importante en las ecuaciones 1 y 2 (ver sección 3), presentó una variación entre 2 y $6 \mathrm{~mm} / \mathrm{d}$ y los valores más altos se observaron en los meses de abril a septiembre. Para su estimación se empleó el método de Penman-Monteith; la descripción detallada de las estimaciones así como los valores de todas las variables usadas puede consultarse en Jiménez et al. (2012). Se pudo observar que existe una correspondencia entre la variación de la temperatura en la zona de estudio y la evapotranspiración. Con la aplicación del factor del cultivo de caña de azúcar $\left(\mathrm{k}_{\mathrm{ca}}\right)$ en las diferentes etapas de su ciclo de crecimiento como se describe por Brouwer \& Heibloem (1986), se observa en la Figura 8 el comportamiento de la evapotranspiración asociada al cultivo. Una vez obtenidos los resultados de la evapotranspiración, se estimó la huella hídrica de la caña de azúcar $(\mathrm{HH})$, considerando un rendimiento del cultivo de 110 ton/ha. Los resultados de la huella hídrica histórica de la caña de azúcar para el período de riego (Figura 7) muestran que varió entre 100 a $107 \mathrm{~m}^{3} /$ ton con un promedio anual de $104.9 \mathrm{~m}^{3} /$ ton.

En la Tabla 4 se muestra la evapotranspiración acumulada del cultivo así como la huella hídrica de la caña de azúcar acumulada en los meses de riego, también se puede observar que la huella hídrica varía mes con mes, siendo febrero el mes que menor huella hídrica tiene.

La huella hídrica de $104.9 \mathrm{~m}^{3} /$ ton es un valor sobreestimado, ya que se estimó considerando que las 15,500 hectáreas se regaron por inundación (rodado), cuando en la región de Tamazula utilizan riego rodado en un $50 \%$ de la superficie cultivada, en $25 \%$ aproximadamente de suelo agrícola se emplea riego por aspersión y en el $25 \%$ restante el riego es por goteo. Si se toman en cuenta estos dos últimos sistemas de riego, el área regada y la proporción de agua que se ahorra con ellos -el ahorro de riego por aspersión en comparación con inundación es del $30 \%$ y por goteo es del $44 \%$ (Narayanamoorthy,

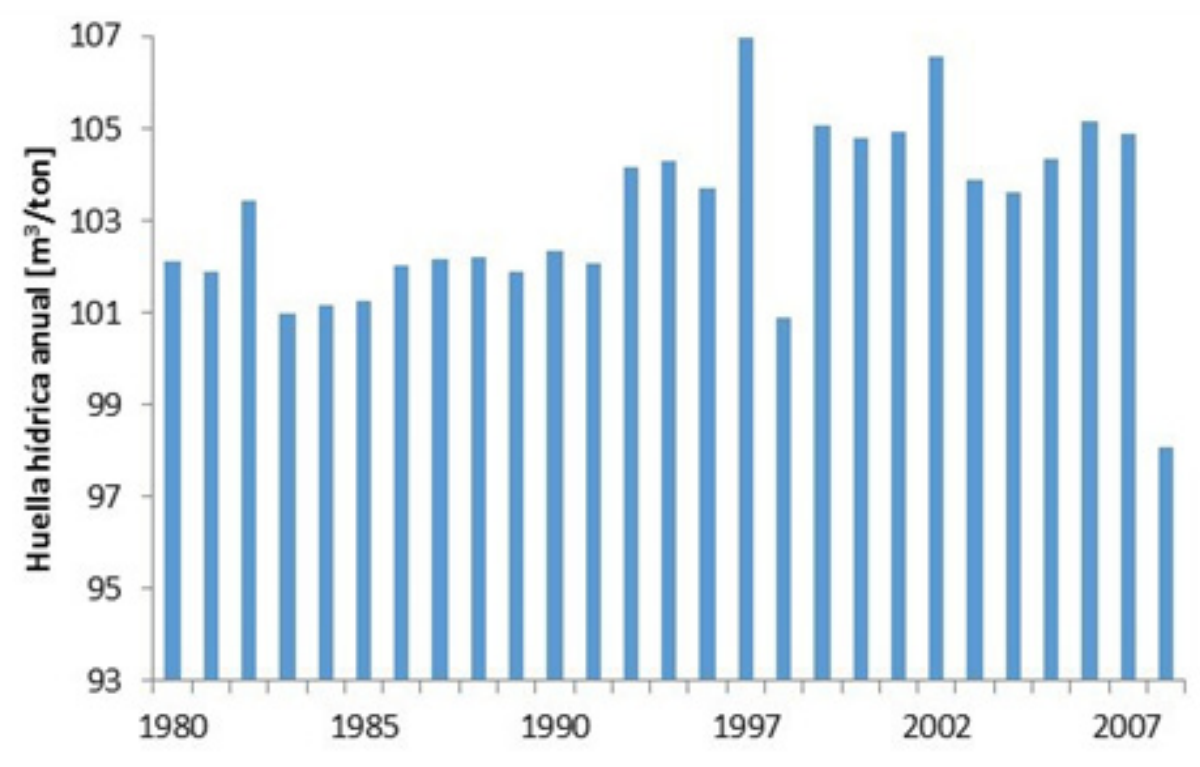

FIGURA 7 - Huella hídrica anual 1980-2007. 
TABLA 4 - Evapotranspiración y huella hídrica histórica de la caña de azúcar estimada.

\begin{tabular}{lrrrr}
\hline Mes & $* \mathbf{K}_{\text {ca }}$ & $\begin{array}{c}\text { Evapotranspiración } \\
\text { acumulada } \\
{[\mathbf{m m}]}\end{array}$ & $\begin{array}{c}\text { Huella } \\
\text { Hídrica } \\
{\left[\mathbf{m}^{3} / \mathbf{t o n}\right]}\end{array}$ & $\begin{array}{c}\text { Huella Hídrica } \\
\text { acumulada } \\
{\left[\mathbf{m}^{3} / \mathbf{t o n}\right]}\end{array}$ \\
\hline Febrero & 0.50 & 65.55 & 5.99 & 5.99 \\
Marzo & 0.650 & 186.76 & 11.45 & 17.44 \\
Abril & 0.90 & 350.79 & 14.99 & 32.43 \\
Mayo & 1.0 & 538.30 & 17.56 & 49.99 \\
Junio & 1.025 & 647.39 & 9.98 & 59.97 \\
Julio & 1.050 & 844.67 & 17.93 & 77.9 \\
Agosto & 0.925 & $1,005.38$ & 15.1 & 93 \\
Septiembre & 0.80 & $1,128.68$ & 11.89 & 104.9 \\
\hline
\end{tabular}

FUENTE: *Brower y Heibloem (1986)

2005)-, el valor de la huella hídrica que se aproxima mejor a las condiciones de cultivo de la caña de azúcar en Tamazula varía entre $58.7 \mathrm{~m}^{3} /$ ton con riego por goteo, $73.4 \mathrm{~m}^{3} /$ ton para aspersión y $104.9 \mathrm{~m}^{3} /$ ton bajo el sistema de riego rodado, considerando el mismo rendimiento para la caña de azúcar. Tomando en cuenta esta variación en el requerimiento de agua, el volumen total estimado para regar las 15,500 ha, durante los 8 meses que dura el ciclo de riego, es de 145.7 millones de $\mathrm{m}^{3}$ de agua. De tal manera que en Tamazula hay un ahorro neto de agua de $18.5 \%$ que representa 33 millones de $\mathrm{m}^{3}$ de agua aproximadamente por los sistemas de riego instalados.

La otra componente importante en la demanda de agua para la producción de biocombustibles a partir de caña de azúcar es la cantidad de agua necesaria para el procesamiento de las melazas para obtener etanol.

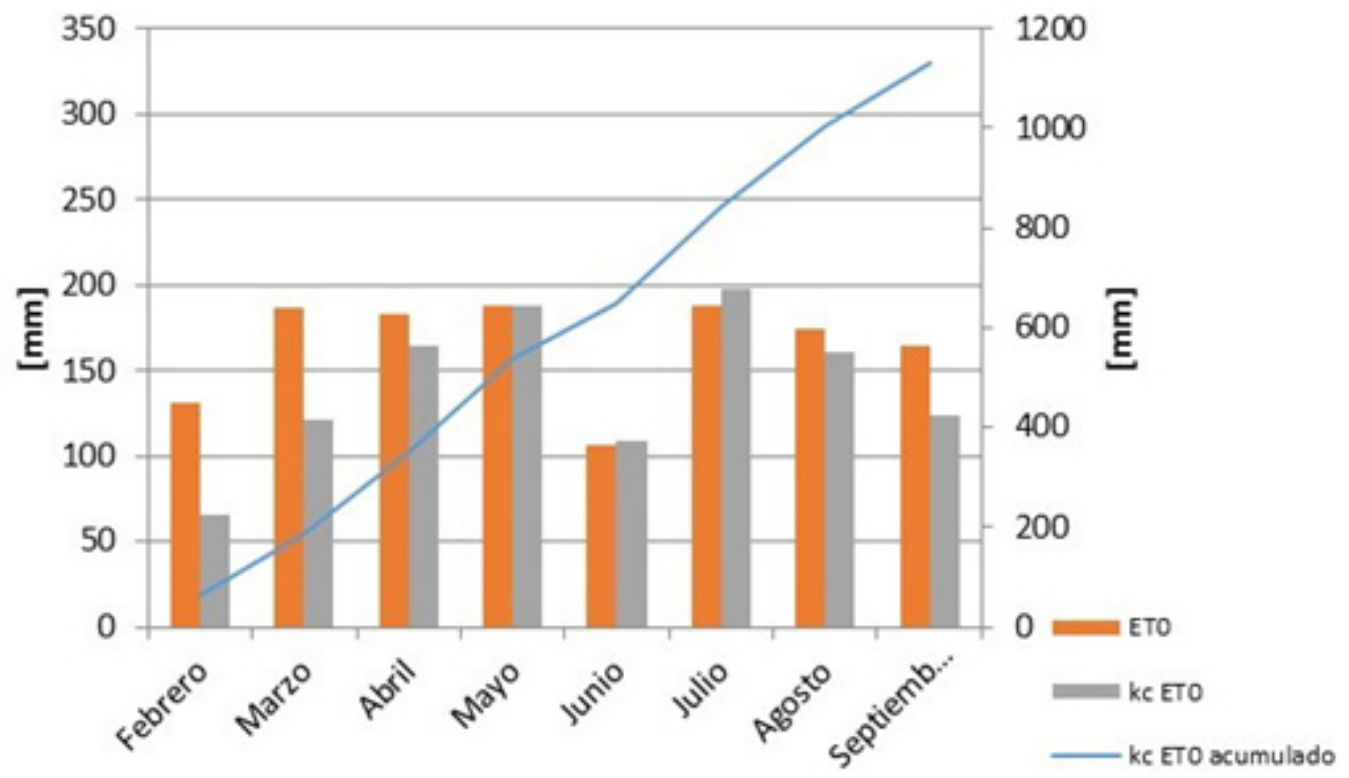

FIGURA 8 - Comportamiento histórico mensual de la evapotranspiración de referencia para la caña de azúcar 
Actualmente, en ninguno de los ingenios del país se produce etanol de forma comercial, sólo se han realizado pruebas piloto, por lo que no hay datos disponibles sobre el consumo de agua para esta etapa de la producción del biocombustible. Por ello, no fue posible medir esta componente de la huella hídrica con datos específicos del ingenio Tamazula. Sin embargo, para tener una estimación se consideraron datos reportados en la literatura con base en el volumen de agua requerido por cada tonelada de caña procesada $\left(1.8 \mathrm{~m}^{3} /\right.$ ton $)$. Con esta tasa de consumo se estimó un volumen de agua de 2,105,301 $\mathrm{m}^{3}$ en total, necesario para el procesamiento en el ingenio Tamazula de las 1,169,612 toneladas de caña de azúcar (UNC, 2011) reportadas para el año 2010. Ese volumen sólo representa el $1.5 \%$ de la demanda de agua estimada para el cultivo de la caña de azúcar bajo las condiciones de riego analizadas.

Se reconoce que la tasa de consumo aplicada podría ser una subestimación, ya que es el resultado de años de experiencia en obtener mejores eficiencias en la industria azucarera brasileña. No obstante, el uso de agua durante la producción de caña es mucho mayor que la necesaria en el proceso de obtención del etanol en los ingenios, por lo que la demanda de agua agrícola es la componente más importante en la estimación de la huella hídrica y por tanto en el impacto en el recurso del agua.

Bajo condiciones de cambio climático es de esperarse que la evapotranspiración y por tanto la demanda de agua para el crecimiento de la caña de azúcar se vean afectadas por cambios en la temperatura y la precipitación, entre otros factores. Por ello, para el cálculo de la huella hídrica futura en la zona cañera de Tamazula, se consideraron proyecciones de precipitación y temperatura para dos horizontes, el año 2020 y 2050. Estos datos climatológicos se obtuvieron de la plataforma
PCIC (2012) correspondientes a los escenarios de cambio climático A2 y B1 y proyectados con los modelos de circulación general ECHAM y GFDL, los cuales fueron seleccionados por que mejor se ajustan al comportamiento histórico del clima en México (Conde et al., 2011). Dichos valores de precipitación y temperatura se usaron en las ecuaciones 1 y 2 , así como en el modelo de Penman-Monteith, para obtener las estimaciones de la huella hídrica. Además, se supuso que prevalecen las condiciones actuales del área cultivada, la tasa de rendimiento y los parámetros locales para estimar la evapotranspiración. El detalle de la investigación puede consultarse en Haro et al. (2013).

Para los escenarios futuros con cambio climático se encontró que la demanda de agua para la producción de caña de azúcar se incrementa de $104.9 \mathrm{~m}^{3} /$ ton a casi $107 \mathrm{~m}^{3} /$ ton (Tabla 5). Estos resultados muestran que si no cambian las condiciones de producción, la huella hídrica se incrementará a futuro entre un 3 y $4 \%$. Este incremento poco significativo puede explicarse si se considera que las proyecciones de los modelos GFDL y ECHAM estiman que se incrementará la precipitación durante los meses de lluvia y disminuirá en el estiaje, de manera que durante los últimos cuatro meses del periodo de riego se contará con mayor cantidad de agua. Estos valores futuros estimados para la huella hídrica de la caña de azúcar se considera que son conservadores ya que podrán presentarse cambios en el rendimiento del cultivo y esperar que la demanda de agua para riego se incremente; por ejemplo la huella hídrica futura podría llegar a ser de $164 \mathrm{~m}^{3} /$ ton, si el rendimiento en Tamazula disminuyera a valores equivalentes al promedio nacional histórico de 72 ton/ha. También puede esperarse que para compensar la caída en el rendimiento se incremente el área cultivada, en cuyo caso la huella hídrica no cambia

TABLA 5 - Huella hídrica y demanda de agua para riego para escenarios con cambio climático.

\begin{tabular}{|c|c|c|c|c|c|c|c|c|}
\hline \multirow{3}{*}{ Año } & \multicolumn{4}{|c|}{$\begin{array}{l}\text { Huella Hídrica futura estimada } \\
\left(\mathrm{m}^{3} / \text { ton }\right)\end{array}$} & \multicolumn{4}{|c|}{$\begin{array}{l}\text { Demanda Total de agua } \\
\text { (Millones } \mathrm{m}^{3} / \mathrm{año} \text { ) }\end{array}$} \\
\hline & \multicolumn{2}{|c|}{ A2 } & \multicolumn{2}{|c|}{ B1 } & \multicolumn{2}{|c|}{ A2 } & \multicolumn{2}{|c|}{$\mathrm{B} 1$} \\
\hline & GFDL & ECHAM & GFDL & ECHAM & GFDL & ECHAM & GFDL & ECHAM \\
\hline 2020 & 107.2 & 107 & 107.2 & 106.4 & 155.6 & 155.2 & 155.6 & 154.5 \\
\hline 2050 & 109 & 109 & 107.9 & 108.7 & 158.1 & 158.1 & 156.5 & 157.7 \\
\hline
\end{tabular}


pero la demanda de agua para riego total aumentará; se estimó que para un $10 \%$ más de hectáreas cultivadas, la demanda de agua para riego crecería en un $8 \%$.

\subsection{Impacto en el recurso hídrico}

Los resultados de esta investigación muestran que la producción del biocombustible etanol a partir de la caña de azúcar presenta una huella hídrica que varía entre 58.7 y $104.9 \mathrm{~m}^{3} /$ ton con una demanda importante de agua en la región de Tamazula del orden de 145.7 millones de $\mathrm{m}^{3}$ por ciclo agrícola. Estas estimaciones que muestran que el mayor consumo de agua está en el riego de la caña (98\%). Sin embargo, hay un ahorro de casi 33 millones de $\mathrm{m}^{3}$ durante cada ciclo agrícola, gracias a que cuentan con instalaciones de sistemas más eficientes de riego, comparados con el tradicional por inundación (rodado).

Un factor importante en estos resultados es la tasa de rendimiento del cultivo de la caña de azúcar en la región (ver Ecuación 1). Si ese rendimiento bajara, por ejemplo de 110 a 100 ton/ha y considerando sólo riego rodado, entonces la huella hídrica de 98.1 se incrementa a $115 \mathrm{~m}^{3} /$ ton; más aún, si se considera el rendimiento promedio histórico del país de 72 ton/ha, la huella hídrica se incrementa hasta $164 \mathrm{~m}^{3} /$ ton. Estos datos indican que el sistema de riego y el rendimiento del cultivo son claves en la demanda de agua y por tanto en el ahorro de la misma.

De tal manera que en Tamazula hay un ahorro neto de casi $20 \%$ que representa aproximadamente 33 millones de $\mathrm{m}^{3}$ de agua por los sistemas de riego instalados. Volumen de agua que no es despreciable ya que esa cantidad es equivalente a dotar $150 \mathrm{~L}$ diarios (Aparicio et al., 2006) a más de 950 mil personas durante los 8 meses de riego o equivalente a dotar de agua potable a siete veces la población del municipio de Tamazula, la cual es cercana a 127,000 habitantes.

Es claro que la metodología de la huella hídrica es una valiosa herramienta que permite analizar los requerimientos de agua para la producción de cultivos, principalmente si se planea destinarla para la producción de biocombustibles; como ocurre en el caso de México. Permite, además, evaluar opciones de ahorro que dependen de la práctica agrícola; sin embargo, la dimensión del impacto en el recurso, como es saber si hay suficiente agua para atender esos requerimientos, queda fuera del alcance de esa metodología. Por ello en esta investigación se realizó un balance hidrológico de carácter general en la región para tener una aproximación a la magnitud del impacto en el recurso. El balance se analizó a partir de estimaciones de la disponibilidad de agua en la región y tres usos consuntivos: uso municipal (el volumen de agua necesario para cubrir la demanda de agua potable para la población en el municipio), uso agrícola (el agua para riego de la caña de azúcar) y el industrial (demanda de agua en el ingenio).

La disponibilidad de agua en una región, o cantidad de agua natural renovable depende de la cantidad de agua de lluvia anual, del volumen que se evapotranspira, y de la que finalmente escurre superficialmente o se infiltra a los acuíferos. De estas variables, la que más impacto tiene en el volumen de agua disponible es la evapotranspiración, ya que se estima que llega a ser del orden del 75\% de la precipitación anual (CONAGUA, 2011).

A partir de los datos de precipitación y temperatura históricos, 2020 y 2050, se estimó la disponibilidad de agua renovable en la superficie del municipio de Tamazula. Los resultados mostraron que la cantidad histórica de agua disponible fue de 255 millones de $\mathrm{m}^{3}$, y para los escenarios futuros con cambio climático es posible que varíe entre 234 y 352 millones de $\mathrm{m}^{3}$ entre el año 2020 y el 2050. Es decir, la posible variabilidad climática podría impactar la reducción del recurso en la región hasta un $8 \%$ en el futuro. Aún cuando es necesario estimar detalladamente el volumen del agua renovable, los resultados indican que es necesario considerar la planeación de los usos del agua de manera integral, incluso a nivel mensual para advertir la probabilidad de escasez temporal o prolongada del recurso.

En cuanto a la estimación de la demanda de los usos consultivos de agua, se obtuvo que para el suministro de agua potable a la población municipal se requiere un volumen de 6.9 millones de $\mathrm{m}^{3}$, suponiendo una dotación de 150 L/hab-día (Aparicio et al., 2006) y una población de 127,000 habitantes. Este volumen, sumado a la demanda de agua para riego de la caña de azúcar (136.3 millones de $\mathrm{m}^{3}$ ) y la correspondiente en el ingenio (2.1 millones de $\mathrm{m}^{3}$ ), indica una demanda total de 154.7 millones de $\mathrm{m}^{3}$ al año, donde el riego representa el 94\% 
de la demanda. Estos valores son representativos para la región en cuanto a que la caña es el principal cultivo, lo cual no excluye la existencia del riego a otros, y también el ingenio es la principal industria en la zona.

El volumen de agua para los usos consuntivos de 154.7 millones de $\mathrm{m}^{3}$ corresponde al $60 \%$ del agua renovable histórica en la región. Este porcentaje, denominado índice de estrés hídrico, indica que la estimación de la tendencia histórica en la región, con un grado de presión alto sobre el recurso ( $>40 \%$, CONAGUA, 2011) es congruente con la clasificación estatal (Fig. 9).

El análisis de estos últimos resultados indica que la tendencia de ir incrementado la extensión de riego por goteo, observada en la zona cañera de Tamazula, es acertada frente a la situación de estrés hídrico en la región, ya que contribuirá a incrementar el ahorro de agua. Más aún, se advierte como una medida relevante, dado que pueden presentarse limitaciones serias a la producción de caña de azúcar ante la posible reducción del recurso en la región hasta un $8 \%$ en el futuro.

Ante el impacto en el recurso agua y la competencia en el uso del suelo para la producción de alimentos asociado a la producción de bioetanol a partir de los cultivos más usados, caña de azúcar o maíz, está presente la alternativa de la producción de biocombustibles de segunda generación. Esta corresponde a la producción a partir de recursos que no compiten con la producción de alimentos y que ofrecen ventajas reales en la reducción de las emisiones de gases efecto invernadero. Tal es el caso de la producción a partir de desechos forestales y agrícolas, las cuales se ha evaluado con prometedoras ventajas, principalmente en los países en desarrollo (Machado, 2010). Hay proyectos que están evaluando la producción y los estudios económicos correspondientes para el procesamiento de biomasa de bagazo y paja de caña de azúcar, bagazo de agave azul, biomasa leñosa,

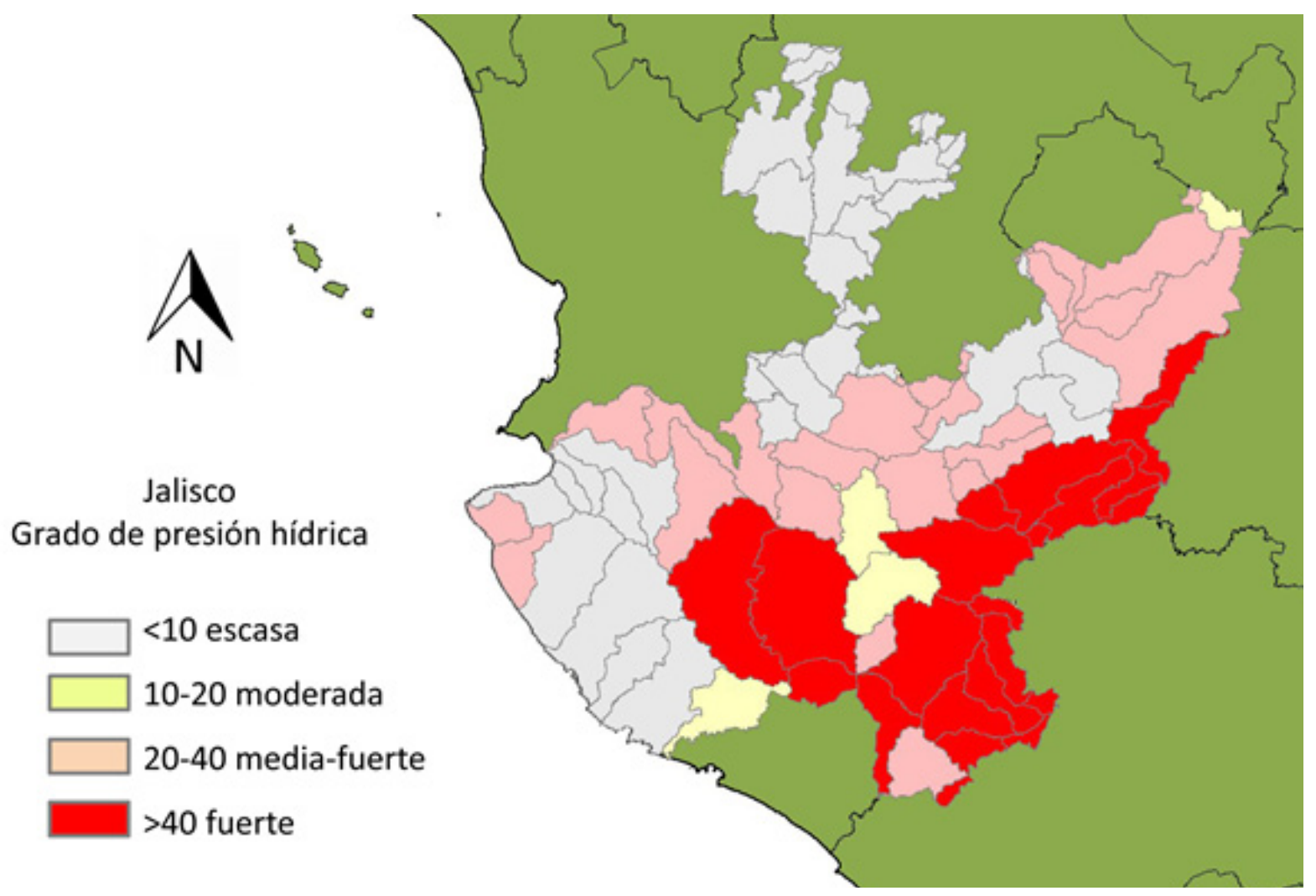

FIGURA 9 - Grado de presión hídrica en el estado de Jalisco (CONAGUA, 2009). 
palma de aceite y cáscara de arroz, entre otras materias primas consideradas (González et al., 2008; IMP, 2009; Hernández-Méndez et al., 2012; Chiaramonti et al., 2013). El desarrollo tecnológico para su procesamiento está aún en proceso, sin embargo se advierte que deberán atenderse las condiciones locales para beneficiar a las economías locales y promover el desarrollo rural (Eisentraut, 2010), ya que la elección de la materia prima dependerá de las condiciones climáticas y edafológicas de cada país.

\section{Reflexión final}

A partir de la experiencia en el caso de estudio reportado en esta investigación, se presentan las siguientes observaciones para futuras investigaciones vinculadas a la planeación de opciones de medidas de mitigación en el país.

- Los resultados para el caso Tamazula no se pueden generalizar a otras zonas cañeras o ingenios del país, ya que las zonas cañeras se localizan en regiones con rasgos distintos en cuanto a la cantidad de agua disponible y presentan diferentes eficiencias de producción. Por lo que es necesario realizar estudios específicos a las condiciones de cada zona para analizar tanto la magnitud de la huella hídrica local como evaluar el balance hídrico entre los usos consuntivos de agua y el comportamiento del volumen de agua renovable actual y futuro

- Cada opción de mitigación orientada a la producción de alternativas a los combustibles de origen fósil para el transporte, como lo es el bioetanol a partir de la caña de azúcar o cualquier otra opción de segunda generación, deberá de contar con el análisis a nivel local de su viabilidad considerando el impacto tanto en la seguridad energética como la seguridad hídrica del país
- Los estudios específicos a nivel local deberán incorporar el análisis de las variables económicas para dimensionar adecuadamente su viabilidad. En el caso concreto de la caña de azúcar es necesaria la definición de una política de precios clara que haga atractiva la producción de bioetanol frente a la comercialización del azúcar y la gasolina

- Se pretendió marcar metas para la sustitución de la gasolina por bioetanol, a partir de caña de azúcar, en el transporte urbano de las zonas metropolitanas de la Ciudad de México, Monterrey y Guadalajara (SENER, 2008); sin embargo, a la fecha sólo se han realizado pruebas a escala piloto de la producción de bioetanol en el país

- La producción de bioetanol para sustituir un porcentaje $(6 \%)$ de gasolina en el transporte urbano como una opción ecológica pareciera más atractiva que como una opción energética y medida de mitigación, frente a los efectos del cambio climático. Sin embargo, debería de evaluarse esta opción y establecer la viabilidad para reducir la emisión de gases contaminantes en las grandes ciudades del país

- Finalmente, los biocombustibles de primera generación, bioetanol y biodiesel a partir de cultivos comerciales, podrían rápidamente ser superados por los denominados biocombustibles de segunda generación. Estos se producen de productos agrícolas y forestales, distintos de los cultivos alimentarios, que aprovechan la lignina, la celulosa y la hemicelulosa de la planta (FAO, 2008). Los avances tecnológicos están evolucionando rápidamente a nivel internacional y México no puede perder esa perspectiva de desarrollo. Por lo que es recomendable invertir en investigaciones sobre la viabilidad de ellos, así como de otras opciones energéticas de mitigación. 


\section{Referencias}

Allen, R. G.; Pereira, L. S.; Raes, D.; Smith, M. Crop Evapotranspiration - Guidelines for Computing Crop Water Requirements. FAO Irrigation and Drainage Paper 56. Rome: Food and Agriculture Organization of the United Nations) 1998.

Aparicio, M. J.; Lafragua, C. J.; Gutiérrez, L. A.; Mejía, Z. R.; Aguilar, G. E. Evaluación de los recursos hídricos. UNESCO, 2006.

Bates, B. C.; Kundzewicz, Z. W.; Wu, S.; Palutikof, J. P. (Eds.). Climate Change and Water. Technical Paper of the Intergovernmental Panel on Climate Change. Geneva: IPCC Secretariat, 2008.

Brouwer, C.; Heibloem, M. Irrigation Water Management: Irrigation Water Needs. Training Manual No. 3. Rome: FAO, 1986.

CEA. Región 05 Sureste. Sistema de Información del Agua. Comisión Estatal del Agua Jalisco. 2011. Disponible en: $<$ http://www.ceajalisco.gob.mx/>. Consultada en: 15/12/2011.

Chiaramonti, D.; Giovannini, A; Janssen, R.; Mergner, R. $2^{\circ}$ Generation Bioethanol The world's largest demo plant ready to be transferred all over the world. WIP Renewable Energies, Munich, Germany, 2013. p. 52.

Chiu, Y.; Walseth, B.; Suh, S. Water Embodied in Bioethanol in the United States. Environmental Science and Technology, 43(8), 2688-2692, 2009.

CONAGUA. Programa hídrico visión 2030 del Estado de Jalisco. Comisión Nacional del Agua. Secretaría del Medio Ambiente y Recursos Naturales, 2009.

Estadísticas del Agua en México 2011. Comisión Nacional del Agua. Secretaría de Medio Ambiente y Recursos Naturales, 2011.

Conde, C.; Estrada, F.; Martínez, B.; Sánchez, O.; Gay, C. Regional climate change scenarios for México. Atmósfera, 24(1), 125-140, 2011.

Coviello, M. F.; Cómez, J. J.; Razo, C.; Rodríguez, A. Biocombustibles líquidos para el transporte en América Latina y el Caribe. CEPAL-GTZ, 2008.

De Fraiture, C.; Giordano, M.; Liao, Y. Biofuels and implications for agricultural water use: Blue impacts of green energy. Water Policy, 10(S1), 67-81, 2008.

Domingo, F.; Villagarcía, L.; Were, A. ¿Cómo se puede medir y estimar la evapotranspiración?: estado actual y evolución. Eco- sistemas, 2003/1. 2002. Disponible en: <http://www.aeet.org/ ecosistemas/031/informe1.htm>. Consultada en: 15/08/2011.

Eisentraut, A. Sustainable production of second-generation biofuels. Potencial and perspectives in major economies and developing countries. Extended Executive Summary. International Energy Agency (IEA). 2010.

Elcock, D. Baseline and Projected Water Demand Data for Energy and Competing Water Use Sectors. Environmental Science Division, Argonne National Laboratory, Oak Ridge, TN: U.S. Department of Energy, 2008.

FAO. Bosques y energía. Cuestiones clave. Roma: Organización de las Naciones Unidas para la Agricultura y la Alimentación, 2008.

. Usos del Agua. AQUASTAT Sistema de información global sobre el agua y la agricultura de la FAO. (2012a). Disponible en: < http://www.fao.org/nr/water/aquastat/water_use/ indexesp.stm>. Consultada en: Marzo 2012.

. Water resources. AQUASTAT FAO's Information System on Water and Agriculture. 2012b. Disponible en: $<$ http:// www.fao.org/nr/water/aquastat/countries_regions/indexesp. stm>. Consultada en: Marzo 2012.

Felix, Erika; Rosell, Cadmo (Eds.). Bioenergía y seguridad alimentaria BEFS. El análisis de BEFS para el Perú. Compendio técnico. Volumen II. Metodologías. FAO - Technical Papers: Ambiente Cambio Climático Bioenergía. Seguimiento y Evaluación. 2010, p. 170.

Fingerman, K. R.; Torn, M. S.; O’Hare, M. H.; Kammen, D. M. Accounting for the water impacts of ethanol production. Environmental Research Letters, 5(10), 1-7, 2010.

Fresco Louise, O. Biomass, food \& sustainability: Is there a dilemma? University of Amsterdam. Member of the Supervisory Board of Rabobank Nederland with Daan Dijk and Wouter de Ridder, Rabobank, The Netherlands, 2007. p. 52. Disponible en: <http:/www.rabobank.com/content/images/ Biomass_food_and_sustainability_tcm43-38549.pdf $>$. Consultada en: 20/05/2012.

Gerbens-Leenes, W.; Hoekstra, A. Y.; Van der Meer, T. H. The water footprint of bioenergy, Proceedings of the National Academy of Sciences, 106(25), p. 10219-10223, 2009.

The water footprint of sweeteners and bioethanol from sugar cane, sugar beet and maize. Value of Water Research Report Series 38, UNESCO-IHE, 2009a. 
Gleick, P. The World's water. Island Press, 2000.

Goettemoeller, J.; Goettemoeller, A. Sustainable Ethanol: Biofuels, Biorefineries, Cellulosic Biomass, Flex-Fuel Vehicles, and Sustainable Farming for Energy Independence. Praire Oak Publishing: Maryville, Missouri, 2007.ISBN 978-0-97862930-4, p. 196.

González, A. F.; Jiménez, I. C.; Rodríguez, S. M.; Restrepo, S.; Gomez, J. M. Biocombustibles de $2^{\text {a }}$ generación y biodiesel. Una mirada a la contribución de la Universidad de los Andes. Revista Ingeniería, 28, 70-82, julio/dic. 2008.

GRFA.Global Renewable Fuels Alliance. 2011. Disponible en: $<$ http://www.globalrfa.org/>.

Haro, M. E.; Navarro, I.; Thompson, R.; Jimenez, B. Estimation of the water footprint of sugarcane in Mexico: is ethanol production an environmentally feasible fuel option? Journal of Climate Change, en prensa.

Hernández-Mendez, O.; Vandenbossche, V.; Brault, J.; Montiel-Pacheco, C.; Vivaldo-Lima, E.; Hernández-Luna, M.; Barzana, E.; Rigal, L.; Vilarem, G. Pre-treatment and enzymatic hydrolysis of blue agave bagasse (Babethanol). Anales del VI Seminario Lationamericano y del Caribe en Biocombustibles, Cuernavaca, México, Agosto 2012. Disponible en: <http://babethanol.ensiacet.fr/IMG/pdf/BABETHANOL_UNAM_presentation_at_Cuernavaca_august_2012.pdf $>$.

Hoekstra, A. Y. Human Appropriation of Natural Capital: Comparing Ecological Footprint and Water Footprint Analysis. Institute for Water Education. Ecological Economics, 68, 1963-1974, 2007.

; Chapagain, A. K. Water footprints of nations: Water use by people as a function of their consumption pattern. Water Resour, Manag., 21, p. 35-48, 2007.

; . Globalization of Water. Sharing the Planet's Freshwater Resources. Oxford, UK: Blackwell Publishing, 2008. ISBN 978-1-4051-6335-4. 224 p.

; Aldaya, M. M.; Mekonnen, M. M. Water Footprint Manual. State of the Art 2009. Enschede, Netherlands: Water Footprint Network, 2009.

; Hung, P. Q. Virtual Water Trade: A Quantification of Virtual Water Flows Between Nations in Relation to International Crop Trade. Value of Water Res Report Series 11. Delft, The Netherlands: UNESCO-IHE, 2002.

Hofstrand, D. Greenhouse gas emissions of corn ethanol production. AgMRC Renewable Energy Newsletter, August, 2009.
Hughes, S.; Partzch, L.; Gaskell, S. The development of biofuels within the context of the global water crisis. Sustain Dev Law \& Policy, 62, 58-62, 2007.

IMP. Alternativas tecnológicas de la producción de biocombustibles en México. 2009. Disponible en: <http://www.imp. $\mathrm{mx} /$ comunicacion $/$ gaceta/?imp $=$ nota\&nota $=090216-1 \mathrm{dfr}>$.

Jank, M. J.; Kutas, G.; do Amaral, L.; Nassar, A. M. EU and U.S. Policies on Biofuels: Potential Impacts on Developing Countries. The German Marshall Fund of the United States. Washington DC, 2007. 28 p. Disponible en: <http://www. gmfus.org/galleries/ct_publication_attachments/GMF_USEU_ Final.pdf>. Consultada en: Mayo 2011.

Jiménez, B.; Navarro, I.; Haro, M.; Thompson R. Evaluación de la disponibilidad de agua y los costos asociados a la producción de cultivos para la manufactura de biocombustibles. Informe Final del Proyecto "Biocombustibles en México: una alternativa para la reducción de la dependencia de los hidrocarburos y para la mitigación de los gases efecto invernadero". Convenio Instituto de Ingeniería-PINC-UNAM, 2012.

Machado, C. M. M. Situación de los Biocombustibles de $2^{a} y$ $3^{a}$ Generación en América Latina y Caribe. OLADE-IICA, Agosto 2010.

Maluenda, G. M. Bioetanol. Perspectivas 2012. 2011. Disponible en: $<$ http://www.agrodigital.com/Documentos/bioetanolmz12.pdf $>$. Consultada en: 02/07/2012.

Mekonnen, M. M.; Hoekstra, A. Y. The green, blue and grey water footprint of crops and derived crop products, Volume 1: Main Report. Value of Water Research Report Series No. 47, Delft, the Netherlands: UNESCO-IHE, 2010.

Moreira, J. R. Water use and impacts due ethanol production in Brazil. 2007 Disponible en: <http://www.iwmi.cgiar. org/EWMA/files/papers/Jose_Moreira.pdf $>$. Consultada en: Abril 2011.

Narayanamoorthy, A. Potential for Drip and Sprinkler Irrigation in India. 2005. Disponible en: <http://nrlp.iwmi. org/.../12.\%20Water\%20Savings\%20Technologies\%20-\%20 Narayanmoorthy.pdf $>$. Consultada en: 20/05/2011.

NRC. Water Implications of Biofuels Production in the United States. National Research Council, Washington, D.C.: National Academies Press, 2008. p 19-25.

PCIC. Pacific Climate Impacts Consortium Regional Analysis Tool (BETA). 2012. Disponible en: < http://pacificclimate.org/ tools-and-data $>$. Consultada en: 13/06/2012. 
Pimentel, D. Ethanol fuels: Energy balance, economics, and environmental impacts are negative. Nat. Resour. Res., 12(2), 127-134, 2003.

Ramos C. L. Análisis de los Efectos de la Producción de Cultivos Bioenergéticos sobre la Disponibilidad de los Recursos Hídricos: El Caso del Sistema Chira. Capítulo 4 en: Felix, Erika; Rosell, Cadmo (Eds.). Bioenergía y seguridad alimentaria BEFS. El análisis de BEFS para el Perú. Compendio técnico. Volumen II. Metodologías. FAO, 2010. p. 53-69. Disponible en: <http://www.fao.org/docrep/013/i1712s/i1712s04.pdf>. Consultada en: 27/10/2012.

REN21 Renewables 2007 Global Status Report. 2008. Disponible en: $<$ http://www.ren21.net $>$.

Roberts, M. G.; Male, T. D.; Toombs, T. P. Potential Impacts of Biofuels Expansion on Natural Resources. A Case Study of the Ogallala Aquifer Region. Environmental Defense. 2007. Disponible en: <http://www.globalwarming.org/wp-content/ uploads/2010/12/edf-study-of-ethanol-water-impacts.pdf $>$. Consultada en: Agosto 2011.

SAGARPA. Estudio de gran visión para la identificación de necesidades de riego y drenaje en las zonas de abasto cañeras y propuestas de tecnificación en zonas potenciales como base para el desarrollo de proyectos de inversión. Etapa I. SAGARPA-PRONAC-SIAP, 2009. p. 83.

Saulino, F. Implicaciones del desarrollo de los biocombustibles para la gestión y el aprovechamiento del agua. Comisión Económica para América Latina y el Caribe CEPAL, 2011.

SENER. Potenciales y Viabilidad del Uso de Bioetanol y Biodiesel para el Transporte en México. Secretaría de Energía (Proyectos ME-T1007 - ATN/DO-9375-ME y PN 04.2148.7001.00). 2006. Disponible en: <http://www.sener.gob.mx/res/ PE_y_DT/pub/Biocombustibles_en_Mexixo_Estudio_Completo.pdf $>$. Consultada en: Febrero 2011.
. Ley de promoción y desarrollo de los bioenergéticos. Secretaría de Energía. 2008. Disponible en: $<$ http://www. diputados.gob.mx/LeyesBiblio/pdf/LPDB.pdf $>$. Consultada en: $11 / 03 / 2011$.

. Recomendaciones de especificaciones técnicas para el etanol y sus mezclas (E6) y la infraestructura para su manejo en México. Secretaría de Energía. 2010 Disponible en: http:// www.energia.gob.mx/res/169/sp_RecomendacionesTecEtanolMezclas.pdf $>$. Consultada en: 15/03/2011.

Shapouri, H.; Gallagher, P. W.; Nefstead, W.; Schwartz, R.; Noe, S.; Conway, R. 2008 Energy Balance for the Corn-Ethanol Industry, USDA. Agricultural Economic Report, 846, 15, 2010.

UNC. Estadísticas de la Agroindustria de la caña de azúcar. Unión Nacional de Cañeros, A.C. CNPR, 2011. Disponible en: <http://www.caneros.org.mx/site_caneros/estadisticas/ ingenios/tamazula.pdf $>$. Consultada en: 07/12/2011.

UNC. Estadísticas de la Agroindustria. Unión Nacional de Cañeros A.C. 2012. Disponible en: < http://www.caneros.org. $\mathrm{mx} /$ estadisticas.html>. Consultada en: Agosto 2012.

Van Meekeren, B. T. The Water Footprint of Bio-energy. Master Thesis Report. Department Water Engineering and Management. Enschede: University of Twente, 2008. Disponible en: $<$ http://essay.utwente.nl/BA20CB7F-8B9E-48C1-BFCFA96C0A2785D6/FinalDownload/DownloadId-5154D99B72857E76C34D82DD7263FF21/BA20CB7F-8B9E-48C1BFCF-A96C0A2785D6/58132/1/scriptie_B_van_Meekeren. pdf>.

Varghese, S. Biocombustibles y desafios globales para el agua. Trade and Global Governance, Institute for Agriculture and Trade Policy (IATP). DKA Austria, 2007 Disponible en: $<$ http://www.iatp.org/files/451_2_105551.pdf >. Consultada en: 21/03/2012. 